\title{
Carcinoembryonic antigen (CEA) in the normal human small intestine: a light and electron microscopic study
}

\author{
P. ISAACSON AND MARY A. JUDD ${ }^{1}$ \\ From the Department of Pathology, Faculty of Medicine, General Hospital, Southampton
}

SUMMARY An immunoperoxidase method for the demonstration of carcinoembryonic antigen (CEA) in tissues was applied to formalin-fixed paraffin embedded and glutaraldehyde-fixed resin embedded sections of normal human small intestine. CEA could easily be demonstrated coating the surface of the small intestine, lining the crypts, and in goblet cells, indicating its presence there in considerable concentration. At the ultrastructural level CEA was localised in the glycocalyx and in mucin granules of goblet cells but not intracytoplasmically.

After the discovery of carcinoembryonic antigen (CEA) by Gold and Freedman (1965) in the serum of patients with colorectal cancer, attention was at first directed to the association of the antigen with colonic disorders. Later, studies of serum levels of CEA were carried out in a wide variety of neoplastic and nonneoplastic diseases of various organs (Martin et al., 1976).

Immunohistochemical demonstration of CEA in tissues has, like the studies of serum levels, been largely confined to the demonstration of antigen in colonic lesions. This is despite the fact that CEA has been successfully extracted from a number of both normal and cancerous tissues (Kupchik and Zamcheck, 1972; Khoo et al., 1973; Pusztaszeri and Mach, 1973; Goldenberg et al., 1976). In the course of an immunohistochemical study of CEA in ulcerative colitis (Isaacson, 1976), strongly positive staining was noted in metaplastic Paneth cells and, after this, in sections of ileum from an ileorectal anastamosis, where the antigen appeared to be present in strong concentration along the mucosal cell border and in goblet cells, as well as in Paneth cell granules. These chance observations led to a formal study of the localisation of CEA in normal human small intestine. During the study the positive staining of Paneth cells proved to be spurious (Isaacson and Judd, 1977) but it became evident that the entire surface of the normal jejunum and ileum is coated with CEA where it stains with an intensity similar to colorectal cancer.

${ }^{1}$ Some of the work described in this paper will form part of a thesis for Fellowship of the Institute of Medical Laboratory Scientists.

Received for publication 28 February 1977
The ease with which CEA could be demonstrated in freely available normal tissue at the light microscope level led to attempts to demonstrate CEA in tissues at the ultrastructural level. Ultrastructually CEA is present in the glycocalyx of the small intestine and in the mucin granules of immature and mature goblet cells.

\section{Methods}

\section{LIGHT MICROSCOPY}

Twenty-seven biopsies of normal adult small intestine (22 jejunal and five ileal) were studied. These were obtained either by peroral biopsy or by resection at laparotomy for disorders not primarily affecting the small intestine-for example, gastroenterostomy for peptic ulcer. Tissues were fixed in formol saline and routinely processed into paraffin blocks; $5 \mu \mathrm{m}$ sections were stained for CEA by the indirect immunoperoxidase technique as described elsewhere (Isaacson and Judd, 1977). Endogenous peroxidase and non-specific cross-reacting glycoproteins were inhibited using $1 \%$ periodic acid. After demonstrating abolition of staining by absorption of antiCEA with purified CEA in two cases, negative controls consisted of a duplicate section stained with substitution of normal goat serum for goat antiCEA. Positive controls, consisting of the same section of colon carcinoma, were included in each staining run.

SEMI-THIN SECTIONS AND ELECTRON MICROSCOPY

Of the 27 cases used for light microscopy portions of six were cut fresh into $1 \mathrm{~mm}^{3}$ cubes and fixed in $5 \%$ glutaraldehyde at $4^{\circ}$ for four to 12 hours. The blocks 786 
were dehydrated in several changes of acetone, infiltrated with an acetone/Spurr resin mixture in a Reichert tissue processr and finally embedded in Spurr resin blocks (Wallis and Griffin, 1973). Tissues were not treated with osmium tetroxide or uranyl acetate at this stage. Sections $(1 \mu \mathrm{m})$ were cut and stained with toluidine blue to locate relevant areas for thin sectioning. These semi-thin sections were also stained for CEA after removal of the resin by etching with sodium methoxide according to the method of Mayor et al. (1961). The staining procedure was the same as that previously described for light microscopy (Isaacson and Judd, 1977) except that the first two steps were omitted.

After the blocks had been trimmed, $60-80 \mathrm{~nm}$ thin sections were cut and collected on nickle grids. These were etched with $5 \% \mathrm{H}_{2} \mathrm{O}_{2}$ and then stained for CEA by floating the grids on the relevant reagents contained in small plastic wells enclosed in a moist chamber. After step 7 of the staining procedure the grids were stained with $1 \%$ osmium tetroxide for 10 minutes and counterstained with lead citrate. Grids were then examined using a Phillips 201 electron microscope.

Controls for semi-thin and thin sections were the same as those used for light microscopy.

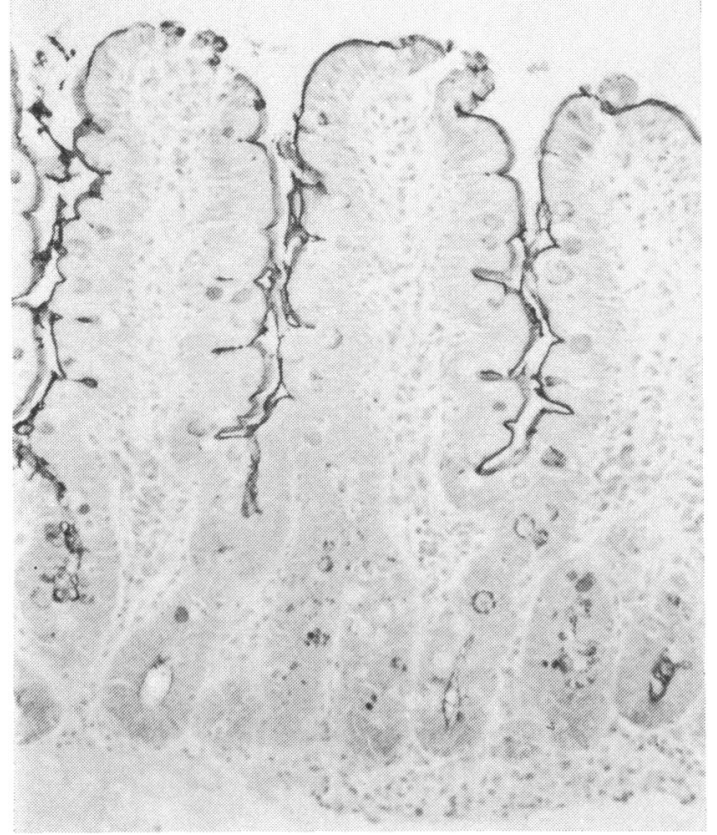

Fig. 1 Formalin-fixed, paraffin embedded section of normal human jejunum stained for CEA with positive staining of the epithelial surface, crypt borders and goblet cells. $\times 100$.

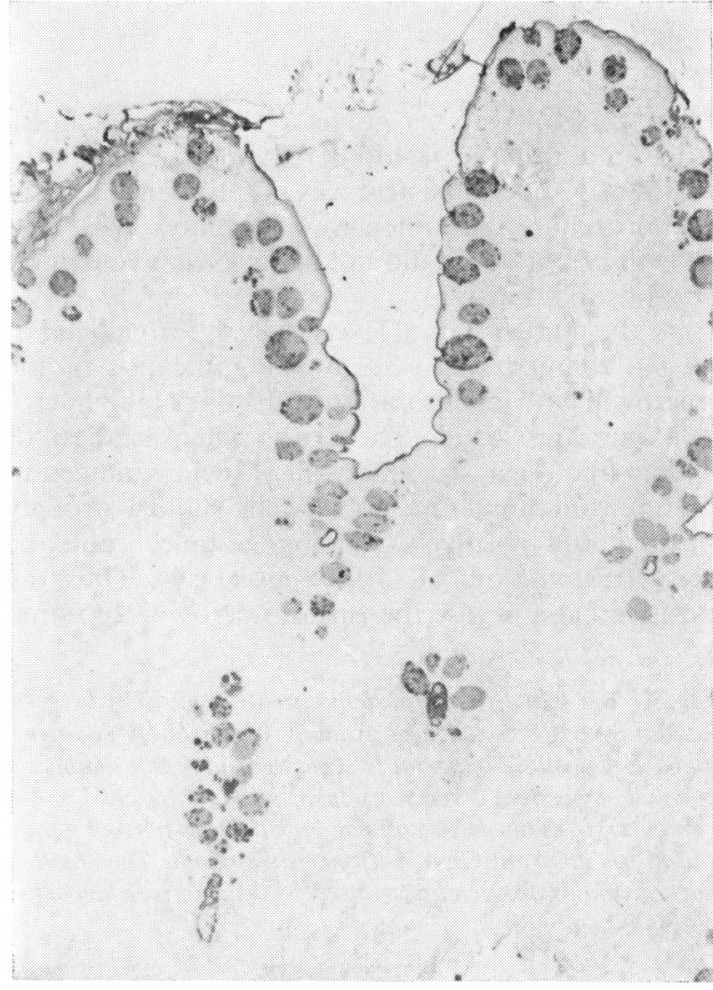

Fig. 2 Glutaraldehyde-fixed, resin embedded $1 \mu \mathrm{m}$ section of normal ileum stained for CEA. Goblet cell staining is much more marked than in paraffin embedded sections. $\times 100$.

\section{Results}

\section{LIGHT MICROSCOPY}

There was strongly positive staining over the surface of villous epithelium, intensifying as a lumenal border in the crypts (Fig. 1). Goblet cells stained variably and in some sections mucous adherent to the mucosa also showed positive staining. The surface staining of epithelial cells persisted into high dilutions of goat antiCEA (up to $\frac{1}{3200}$ ) P Positive control sections of colon carcinoma stained as previously described with staining persisting up to a $\frac{1}{3200}$ dilution of goat antiCEA. No staining was observed in negative controls.

\section{SEMI-THIN SECTIONS AND ELECTRON} MICROSCOPY

In semi-thin $(1 \mu \mathrm{m})$ sections the surface of the brush border of the villi stained strongly for CEA with intensification along the lumenal border of crypts. In 
Fig. 3 High power view of same section illustrated in Fig. 2. Note the granular staining of goblet cells. CEA is present as a band along the surface of the brush border. $\times 100$.

these thinner sections goblet cells always stained, often in a granular fashion (Figs. 2, 3). Despite the fact that $1 \%$ periodic acid was not used in the staining procedure, endothelium, red blood cells, and Paneth cell granules did not stain. Negative control sections did not stain.

At the ultrastructural level (Figs. 4, 5, 6) a band of strong staining was evident along the tips of the microvilli with incorporation of the tips of the microvilli into this band. There was intensification of staining between the microvilli. Mucin granules in mature and immature goblet cells stained strongly positive and positively staining granules could be seen streaming out of surface goblet cells. Thus, the positive band across the tips of microvilli appeared

Fig. 4 (a) Ultrastructural demonstration of CEA in jejunal mucosa. Goblet cell granules are strongly positive and CEA positive material is seen streaming out of the cell. CEA positive glycocalyx fuses with goblet cell contents. (b) Same area stained, substituting normal goat serum for goat antiCEA, with negative result. The small dark granules are artefact caused by lead citrate deposits. $\times 9000$.

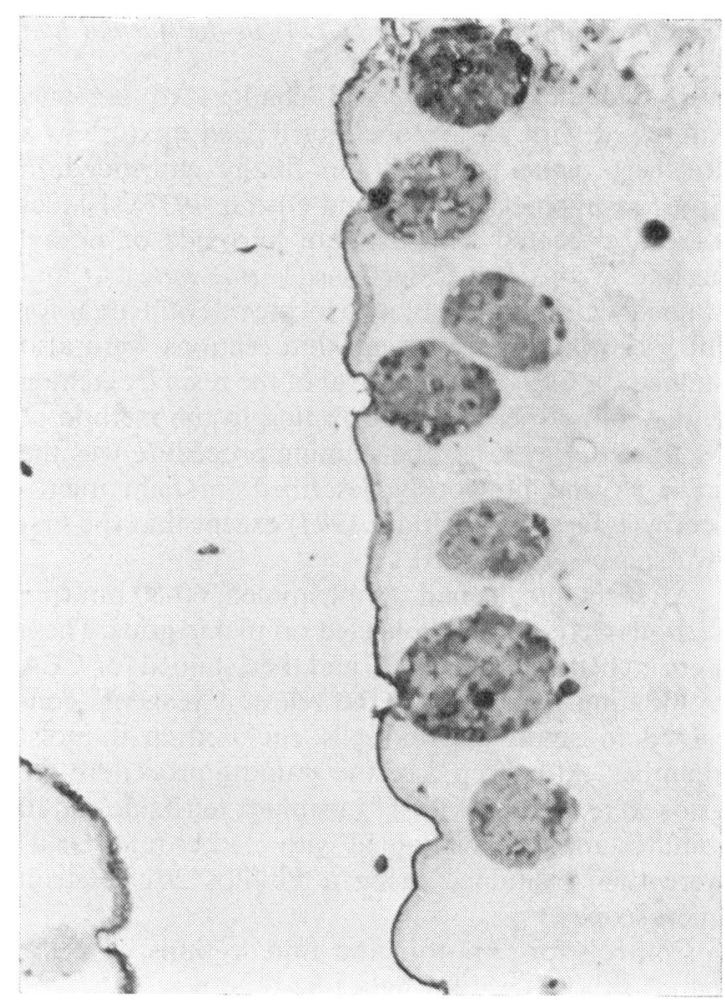

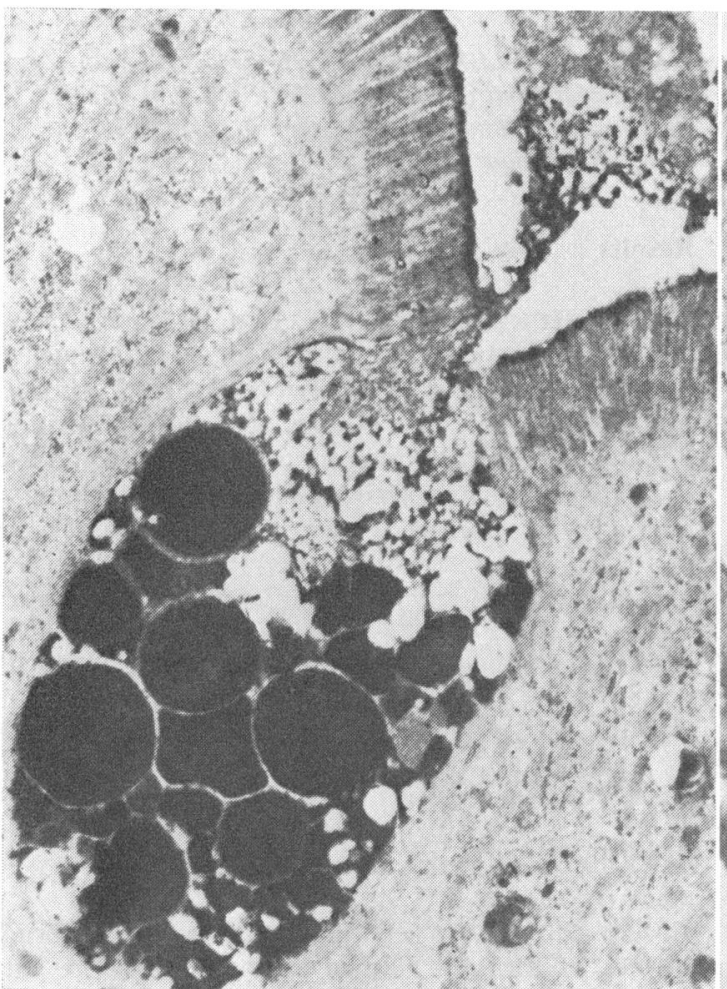

(a)

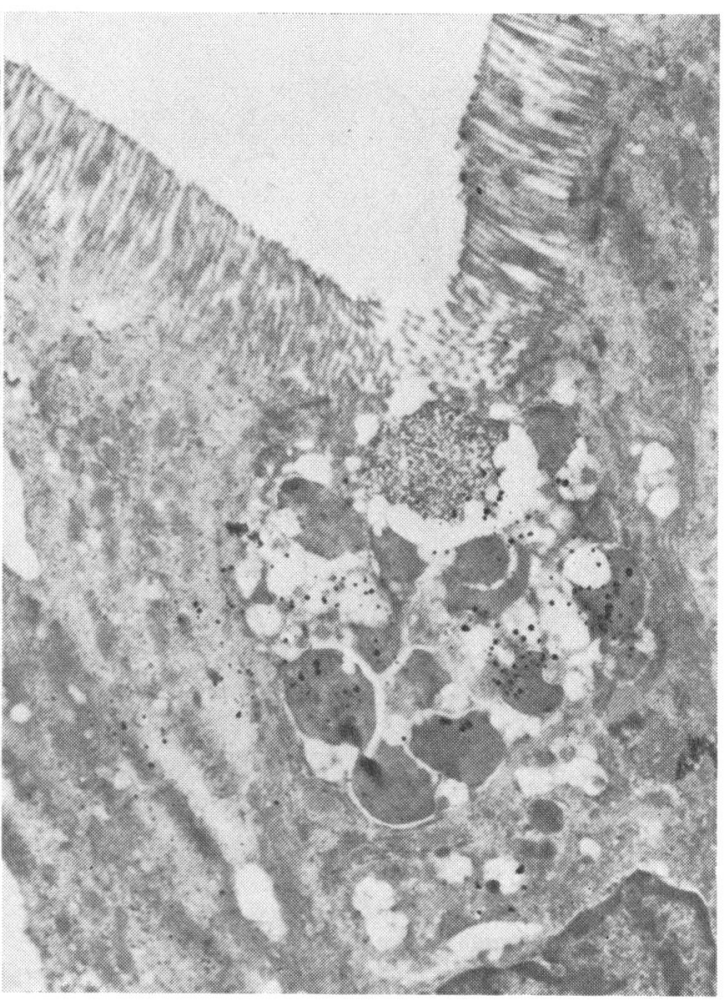

(b) 


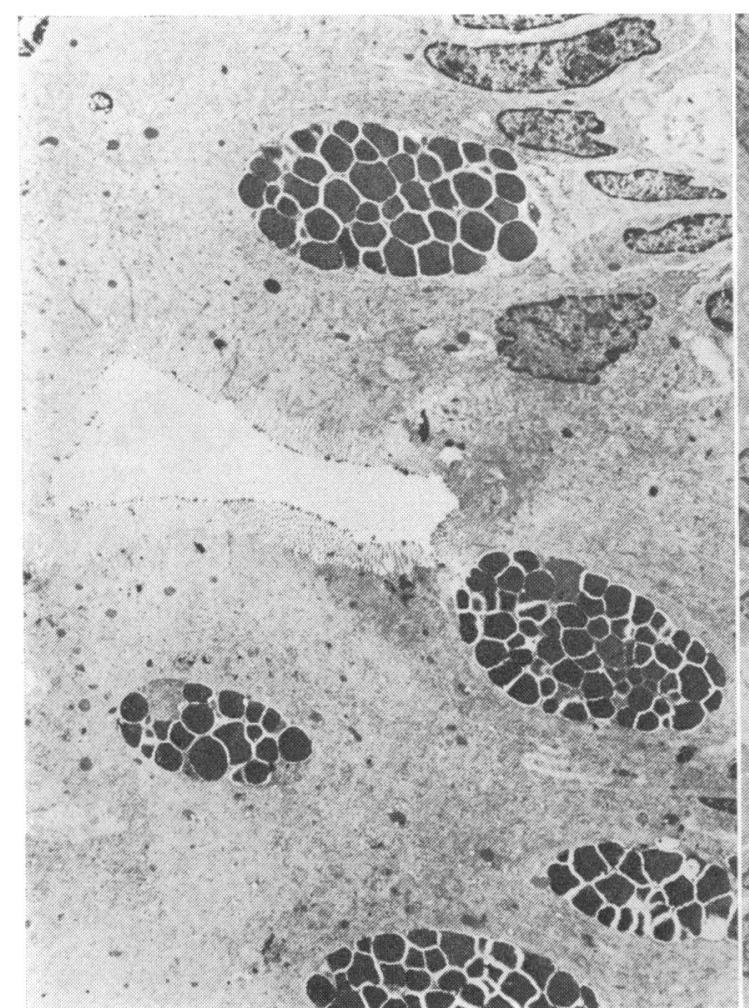

(a)

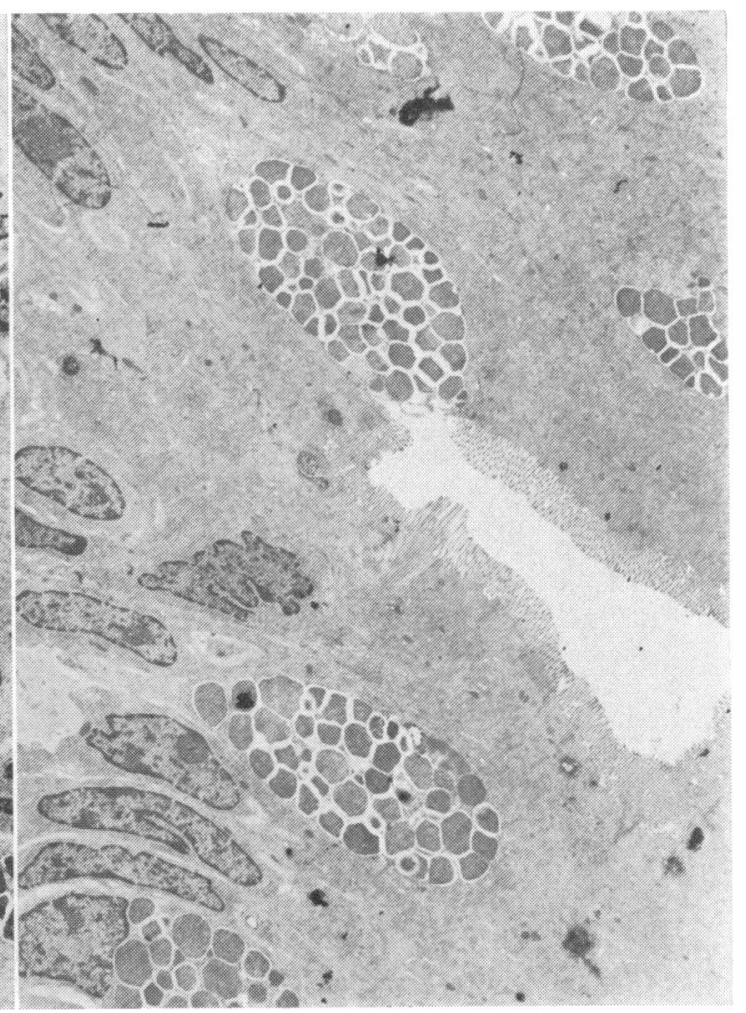

(b)

Fig. 5 (a) Intestinal crypt showing less mature goblet cells staining positively for CEA with positive staining of glycocalyx. (b) Control section stained with normal goat serum is negative. $\times 2500$.

to be largely separate from material escaping from goblet cells, although some fusion of positive material was evident. No intracytoplasmic staining was evident in any of the epithelial cells and, as in the semi-thin sections, no endothelial, red blood cell, or Paneth cell staining was observed. In grids using normal goat serum there was no staining of the goblet cells or the tips of the microvilli. A faint band incorporating the tips of the microvilli could be appreciated.

\section{Discussion}

CEA has previously been demonstrated immunohistochemically in a number of epithelial malignancies, including colorectal cancer, and in normal colon (Goldenberg et al., 1976; Huitric et al., 1976; Isaacson, 1976). It has also been extracted from these and some other normal tissues (Khoo et al., 1973; Goldenberg et al., 1976). While CEA has been demonstrated in foci of intestinal metaplasia in the stomach (Burtin et al., 1973) there have been no previous attempts to demonstrate CEA in the normal human small intestine. This is surprising when the large amount of CEA present in human faeces is considered. Not only is it unlikely that this amount of CEA is derived from the small amounts shown to be present in colonic epithelium (Khoo et al., 1973), but, if it were, then the presence of colonic carcinoma, which contains 10-100 $\times$ more CEA than normal colon, should raise faecal levels of CEA, but, as shown by Elias et al. (1974) this is not the case. Furthermore, Elias et al. found that the CEA content from an ileostomy drainage was equal to that in faeces from normal patients. Additional evidence for the production of CEA by small intestinal epithelium is found in a study of patients with bladder carcinoma. Hall et al. (1973) noted that the urine from ileal conduits contained large amounts of CEA as compared with urine samples taken directly from the ureters of these patients. The ease with which CEA can be demonstrated in the small intestine is yet another indication of the high CEA content of the small intestine since Goldenberg et al. (1976) have shown that the ease with which CEA can be demonstrated immunohistochemically is proportional to 


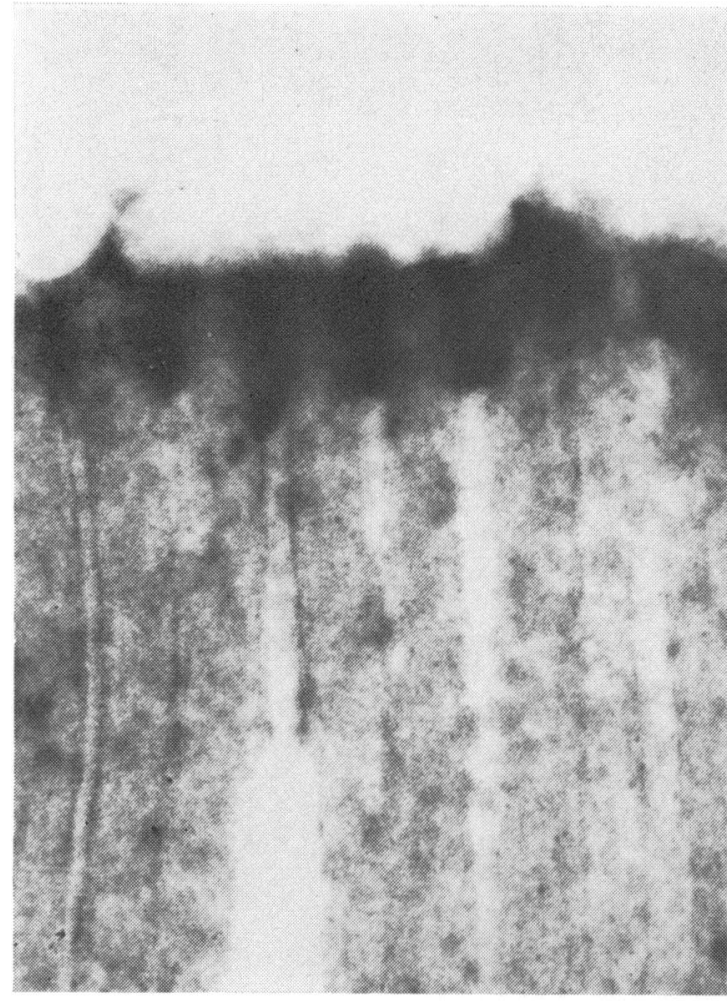

(a)

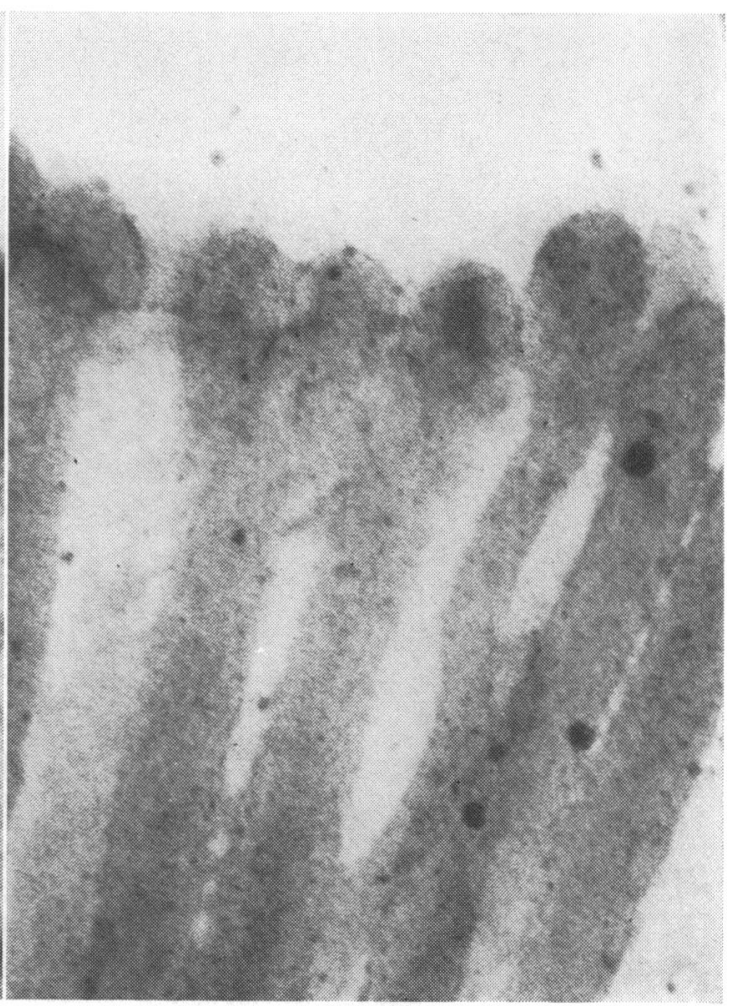

(b)

Fig. 6 (a) Microvilli of jejunum showing incorporation of the tips into a CEA positive band. There is intensification of staining between the villi. (b) Control section stained with normal goat serum. A faint electron dense band incorporates the tips of the microvilli. $\times 112000$.

the tissue concentration. The present study, therefore, indicates that the epithelium of the small intestine is manufacturing CEA in amounts similar to that of colorectal cancer, but, unlike CEA from colorectal cancer, some of which finds its way into the blood stream, small intestinal CEA is mostly shed into the lumen of the gut. Given that the entire adult human small inestine is so well coated with CEA, this can hardly be designated an oncofetal antigen. More probably, as has already been suggested by others (Neville and Laurence, 1974), CEA is related to cell division in some way, being associated with rapidly dividing epithelial tissues. Further study on the biological functions of CEA would seem to be indicated.

CEA has been previously demonstrated in semithin and thin sections of normal colon and colonic carcinoma (Huitric, 1973; Huitric et al., 1976). The methods used have been extremely complex, employing special fixatives and, for ultrastructural studies, blocks were prestained as frozen sections before being processed for electron microscopy. For these studies undiluted antisera were necessary and the results, while in some ways similar to those of the present study, contained some surprising differences, not the least being the negative staining of colonic carcinoma in light microscope preparations. The methods used in our investigation of the small intestine are extremely simple and reproducible. We feel that this is principally because we used Nakane and Kawaoi's (1974) method for preparing peroxidase conjugates, as we found that when we used commercially supplied conjugates, prepared by glutaraldehyde coupling, the sensitivity dropped off dramatically.

It is interesting that the preparation of semi-thin and thin sections inhibited the cross-reaction of antiCEA with other tissue glycoproteins as described previously (Isaacson and Judd, 1977). It was, in fact, this observation that led to the formal study of the immunohistochemical cross-reactivity of CEA. Reagents used for etching the resin were tested and found not to inhibit these reactions. Thus the process of resin embedding had the same effect as pretreating sections with $1 \%$ periodic acid. As no single 
reagent used in resin embedding was found to act in this way we must assume that some combination of reagents or resin polymerisation was responsible.

The glycocalyx is usually described as a fuzzy layer above the microvilli (Toner et al., 1971). No such layer was present in our ultrastructural preparations and instead a band appeared to incorporate the tips of the microvilli. We feel that this can only be the glycocalyx and that its slightly different position is caused by the different preparation and subsequent manipulation of the thin sections. Previous studies, using radiolabelled carbohydrates (Bennett, 1970; Bennett and Leblond, 1970) have indicated that the glyco-protein destined for the glycocalyx is synthesised in the Golgi apparatus from which it migrates into its final position. The restriction of CEA staining to the goblet cell and glycocalyx suggests an alternative possibility-namely, that the glycocalyx, or part of it, is derived from the goblet cell. We have been unable to demonstrate true intracytoplasmic CEA, the glycocalyx and mucin granule being, in effect, extracellular structures. At the light microscope level, CEA has been shown to be present intracytoplasmically in medullary carcinoma of the thyroid (Isaacson and Judd, 1976) and ultrastructural studies of this tumour may be more rewarding in establishing the site of CEA synthesis.

We are indebted to Professor D. H. Wright for his advice and to Mrs Olive Huber for secretarial assistance.

\section{References}

Bennett, G. (1970). Migration of glycoprotein from golgi apparatus to cell coat in the columnar cells of the duodenal epithelium. Journal of Cell Biology, 45, 668-673.

Bennett, G., and Leblond, C. P. (1970). Formation of cell coat material for the whole surface of columnar cells in the rat small intestine, as visualized by radioautography with 1-Fucose- ${ }^{3}$ H. Journal of Cell Biology, 46, 409-416.

Burtin, P., von Kleist, S., Sabine, M. C., and King, $\mathbf{M}$. (1973). Immunohistological localization of carcinoembryonic antigen and non-specific cross-reacting antigen in gastrointestinal normal and tumoral tissues. Cancer $R e-$ search, 33, 3299-3305.

Elias, E. G., Holyoke, E. D., and Chu, T. Ming. (1974). Carcinoembryonic antigen (CEA) in feces and plasma of normal subjects and patients with colorectal carcinoma. Diseases of Colon and Rectum, 17, 38-41.

Gold, P., and Freedman, S. O. (1965). Specific carcinoembryonic antigens of the human digestive system.
Journal of Experimental Medicine, 122, 467-481.

Goldenberg, D. M., Sharkey, R. M., and Primus, F. J. (1976). Carcinoembryonic antigen in histopathology: immunoperoxidase staining of conventional tissue sections. Journal of the National Cancer Institute, 57, 11-22.

Hall, R. R., Laurence, D. J. R., Neville, A. M., and Wallace, D. M. (1973). Carcinoembryonic antigen and urothelial carcinoma. British Journal of Urology, 45, 88-92.

Huitric, E. (1973). An ultrastructural study of the localization of the carcinoembryonic antigen in adenocarcinomas of the human colon. Annales d'Immunologie (Institut Pasteur), $124 \mathrm{C}, 603-608$.

Huitric, E., Laumonier, R., Burtin, P., von Kleist, S., and Chavanel, G. (1976). An optical and ultrastructural study of the localization of carcinoembryonic antigen (CEA) in normal and cancerous human rectocolonic mucosa. Laboratory Investigation, 34, 97-107.

Isaacson, P. (1976). Tissue demonstration of carcinoembryonic antigen (CEA) in ulcerative colitis. Gut, 17, 561567.

Isaacson, P., and Judd, M. A. (1976). Carcinoembryonic antigen in medullary carcinoma of thyroid. Lancet, 2, 10161017.

Isaacson, P., and Judd, M. A. (1977). Immunohistochemistry of carcinoembryonic antigen: characterisation of crossreactions with other glycoproteins. Gut. 18, 779-785.

Khoo, S. K., Warner, N. L., Lie, J. T., and MacKay, I. R. (1973). Carcinoembryonic antigenic activity of tissue extracts: quantitative study of malignant and benign neoplasms, cirrhotic liver, normal adult and fetal organs. International Journal of Cancer, 11, 681-687.

Kupchik, H. Z., and Zamcheck, N. (1972). Carcinoembryonic antigen(s) in liver diseases. Isolation from human cirrhotic liver and serum and from normal liver. Gastroenterology, 63, 95-101.

Martin, E. W. Jr., Kibbey, W. E., DiVecchia, L., Anderson, G., Catalano, P., and Minton, J. P. (1976). Carcinoembryonic antigen, clinical and historical aspects. Cancer, 37, 62-81.

Mayor, H. D., Hampton, J. C., and Rosario, B. (1961). A simple method for removing the resin from epoxyembedded tissue. Journal of Biophysical and Biochemical Cytology, 9, 909-910.

Nakane, P. K., and Kawaoi, A. (1974). Peroxidase-labeled antibody. A new method of conjugation. Journal of Histochemistry and Cytochemistry, 22, 1084-1091.

Neville, A. M., and Laurence, D. J. R. (1974). Report of the workshop on the carcinoembryonic antigen (CEA): the present position and proposals for future investigation. International Journal of Cancer, 14, 1-18.

Pusztaszeri, G., and Mach, J-P. (1973). Carcinoembryonic antigen (CEA) in non-digestive cancerous and normal tissues. Immunochemistry, 10, 197-204.

Toner, P. G., Carr, K. E., and Wyburn, G. M. (1971). The Digestive System-An Ultrastructural Atlas and Review. Butterworth: London.

Wallis, M. A., and Griffin, R. L. (1973). A routine method for embedding animal tissues in Spurr resin for electron microscopy. Journal of Clinical Pathology, 26, 77-78. 